\section{POT1-interacting protein PIP1: a telomere length regulator that recruits POT1 to the TIN2/TRF1 complex}

\author{
Jeffrey Zheng-Sheng Ye, ${ }^{1,3,4,5}$ Dirk Hockemeyer, ${ }^{1}$ \\ Andrew N. Krutchinsky, ${ }^{2}$ Diego Loayza, ${ }^{1}$ Sarah \\ M. Hooper, ${ }^{1}$ Brian T. Chait, ${ }^{2}$ and Titia de \\ Lange ${ }^{1,6}$ \\ ${ }^{1}$ Laboratory for Cell Biology and Genetics and ${ }^{2}$ Laboratory for \\ Mass Spectrometry and Gaseous Ion Chemistry, The \\ Rockefeller University, New York, New York 10021, USA; \\ ${ }^{3}$ Department of Medicine, Memorial Sloan-Kettering Cancer \\ Center, New York, New York 10021, USA
}

Human telomere length is controlled by a negative feedback loop based on the binding of TRF1 to doublestranded telomeric DNA. The TRF1 complex recruits POT1, a single-stranded telomeric DNA-binding protein necessary for cis-inhibition of telomerase. By mass spectrometry, we have identified a new telomeric protein, which we have named POT1-interacting protein 1 (PIP1). PIP1 bound both POT1 and the TRF1-interacting factor TIN2 and could tether POT1 to the TRF1 complex. Reduction of PIP1 or POT1 levels with shRNAs led to telomere elongation, indicating that PIP1 contributes to telomere length control through recruitment of POT1.

Supplemental material is available at http://www.genesdev.org.

Received April 26, 2004; revised version accepted May 14, 2004.

In telomerase-positive human cells, telomere length is influenced by a protein complex, built on the telomeric DNA-binding protein TRF1 (for review, see Smogorzewska and de Lange 2004). The TRF1 complex contains a TRF1-interacting protein, TIN2, and POT1, a protein with an $\mathrm{N}$-terminal $\mathrm{OB}$ fold that confers binding to single-stranded TTAGGG repeats (Chong et al. 1995; Kim et al. 1999; Baumann and Cech 2001; Loayza and de Lange 2003; Loayza et al. 2004). The amounts of TRF1, TIN2, and POT1 are increased on longer telomeres (Smogorzewska et al. 2000; Loayza and de Lange 2003), consistent with a "protein-counting" model of telomere length control (Marcand et al. 1997). When TRF1 or TIN2 is inhibited with mutant alleles or RNAi, telomeres become overelongated by telomerase, indicating that cells measure telomere length based on the amount of TRF1 complex present (van Steensel and de Lange

[Keywords: Telomere; telomerase; TRF1; TIN2; POT1; PIP1]

${ }^{4}$ Present address: Division of Hematology, Departments of Medicine and Pharmacology, New York University School of Medicine, 550 First Avenue, New York, NY 10016, USA.

Corresponding authors.

${ }^{5}$ E-MAIL jeffrey.ye@med.nyu.edu; FAX (212) 263-8444.

${ }^{6}$ E-MAIL delange@mail.rockefeller.edu; FAX (212) 327-7147.

Article published online ahead of print. Article and publication date are at http://www.genesdev.org/cgi/doi/10.1101/gad.1215404.
1997; Kim et al. 1999; Ye and de Lange 2004). A POT1 mutant $(\mathrm{POT} 1 \Delta \mathrm{OB})$ that is incapable of binding to single-stranded telomeric DNA because of deletion of the OB fold can still associate with telomeres through the TRF1 complex. However, this mutant abrogates telomere length control, leading to unopposed telomere elongation by telomerase (Loayza and de Lange 2003). Based on these data, it was proposed that POT1 transduces information on the length of telomeres to the telomere terminus, where telomerase regulation takes place. The larger amounts of TRF1 complex on long telomeres is proposed to increase the number of POT1 molecules able to bind the single-stranded telomeric DNA, allowing POT1 to inhibit telomerase in a manner that is influenced by telomere length.

Because our initial tests for direct interactions of POT1 with TRF1 and TIN2 were negative, we performed mass spectrometry on the TRF1/TIN2 complex to identify proteins that could be responsible for the tethering of POT1. Here we describe a novel component of the TRF1 complex, the POT1-interacting protein PIP1, and document its role in telomere length control. The data further corroborate the role of POT1 as a negative regulator of telomere length and indicate that PIP1 functions to recruit POT1 to the telomeric chromatin.

\section{Results and Discussion}

Mass spectrometric analysis

of the TRF1/TIN2 complex

To isolate new components of the TRF1 complex, TRF1 and TIN2 were tagged with a Flag-HA-HA (FH2) cassette and expressed in HeLa S3 cells by retroviral transduction. Pilot experiments using cotransfection and immunoprecipitation indicated that C-terminally tagged TRF1 (C-FH2-TRF1) and N-terminally tagged TIN2 (N-FH2TIN2) were the most efficient constructs to study complex formation by coimmunoprecipitation (data not shown). Differential salt extraction of HeLa cell chromatin showed that overexpression of TRF1 resulted in an increased loading of TIN2 on chromatin (Supplementary Fig. 1A). Doubly infected HeLaS3 clones were screened for TRF1/TIN2 expression levels, and cell cultures were expanded from a HeLa S3 clone that expressed tagged TRF1 and TIN2 to levels $\sim 5$ - to 10-fold above that of the endogenous proteins (Supplementary Fig. 1A).

The TRF1/TIN2 complex was isolated from $\sim 2 \times 10^{10}$ cells by sequential binding to anti-Flag and anti-HA affinity resin and peptide elution. Tryptic peptides derived from gel-fractionated proteins were identified by MALDI-QqTOF mass spectrometry, and fragmentation spectra of all discernable peptides were obtained using MALDI-ion trap mass spectrometry (Krutchinsky et al. 2000, 2001; see Materials and Methods for details). As expected, the TRF1/TIN2 complex contained TRF1, TIN2, and POT1 (data not shown). Peptides derived from tankyrase 1 and 2, PINX1, or Ku70/86 were not detected. One protein not previously known to occur in the TRF1 complex was identified based on 10 peptides (Supplementary Fig. 1B). Based on the data presented below, we propose to name this protein, which is listed in GenBank as a protein of unknown function (human protein 24432/ BC016904), POT1-interacting protein 1 (PIP1). PIP1 is 
Ye et al.

Table 1. Two-hybrid interaction of PIP1 with TIN2 and POT1

\begin{tabular}{lcc}
\hline & pACT2 $(\mathrm{GAD})$ & PIP1 ::GAD \\
\hline pBTM116 (LexA DBD) & $0.4 \pm 0.2$ & $0.5 \pm 0.06$ \\
POT1 :: LexA & $3.9 \pm 0.05$ & $148 \pm 17$ \\
TIN2:: LexA & $0.04 \pm<0.006$ & $418 \pm 30$ \\
TRF1 : LexA & $0.46 \pm 0.06$ & $1.7 \pm 0.2$ \\
TRF2 : LexA & $0.07 \pm 0.05$ & $0.04 \pm 0.006$ \\
hRap $1:$ LexA & $0.1 \pm 0.006$ & $0.1 \pm 0.006$ \\
\hline
\end{tabular}

Interactions are expressed in $\beta$-galactosidase (Miller) units for the indicated two-hybrid combinations. The numbers represent average values from three independent transformants and standard deviations.

predicted to be a 544 -amino acid/58-kDa protein with no known domains or sequence motifs. PIP1 orthologs are present in the genomes of mouse, rat, and Fugu (Supplementary Fig. 1C). Highly conserved PIP1 genes are not present in the sequenced genomes of insects, worms, or fungi. Queries of the mouse and human EST databases indicate that PIP1, like other components of the TRF1 complex, is widely expressed.

\section{PIP1 is a telomeric protein that tethers} POT1 to TIN2

The interaction of PIP1 with components of the TRF1 complex was addressed by two-hybrid interaction (Table 1). These experiments revealed that PIP1 can bind to both POT1 and TIN2, whereas no interaction was detected between PIP1 and TRF1, TRF2, or hRap1.

Cotransfection into $293 \mathrm{~T}$ cells was used to further analyze the interactions of PIP1 with TIN2 and POT1 (Fig. 1A-D). Cotransfection of tagged POT1 and PIP1 resulted in efficient recovery $(5 \%-10 \%)$ of PIP1 in the POT1 IP, confirming the interaction between POT1 and PIP1 (Fig. 1A). As a control, POT1 failed to coimmunoprecipitate exogenous TIN2. In addition to the interactions between PIP1 and POT1, PIP1 interacted with TIN2. PIP1 was recovered in association with tagged TIN2 (Fig. 1A) and TIN2 could be coimmunoprecipitated with tagged PIP1 (Fig. 1B). Furthermore, a complex containing POT1, PIP1, and TIN2 could be recovered in immunoprecipitates of either POT1 or TIN2 (Fig. 1A,B). This would suggest that PIP1 can link POT1 to TIN2. However, TIN2 IPs also contained POT1 in the absence of PIP1 (Fig. 1A). Because POT1 and TIN2 do not interact in a yeast two-hybrid assay (data not shown), this recovery of POT1 was probably caused by tethering by the endogenous PIP1 in $293 \mathrm{~T}$ cells.

Coimmunoprecipitation experiments indicated that PIP1 did not interact with TRF1 (Fig. 1B). However, when TRF1, TIN2, and PIP1 were cotransfected, both TIN2 and TRF1 could be recovered in the PIP1 immunoprecipitate (Fig. 1B,C), indicating that TIN2 mediated the interaction between PIP1 and TRF1. An N-terminal truncation mutant of TIN2, TIN2-13, which retains the

E
TRF1-interacting domain (Kim et al. 1999), lacked the ability to coimmunoprecipitate PIP1 (Fig. 1C). Consistent with the role of TIN2 in tethering PIP1 to TRF1, this mutant did not lead to recovery of PIP1 in the TIN2TRF1 complex (Fig. 1C).

The POT1 $\triangle \mathrm{OB}$ mutant, which lacks the singlestranded DNA-binding domain, is recruited to telomeres by the TRF1 complex (Loayza and de Lange 2003). This predicts that the PIP1-POT1 interaction is not abolished by deletion of the OB fold of POT1. In agreement, both full-length POT1 and POT1 $1 \mathrm{OB}$ were recovered in IPs of tagged PIP1 (Fig. 1D).

The data on the interaction between the TRF1 complex and PIP1 predict that PIP1 can localize to telomeres. This was addressed by transfection of HeLa1.2.11 cells with Flag-PIP1 and dual immunofluorescence with the Flag antibody and antibodies to endogenous TRF1 or TIN2. The IF data showed that PIP1 primarily localized to telomeres, forming a punctate pattern that coincides
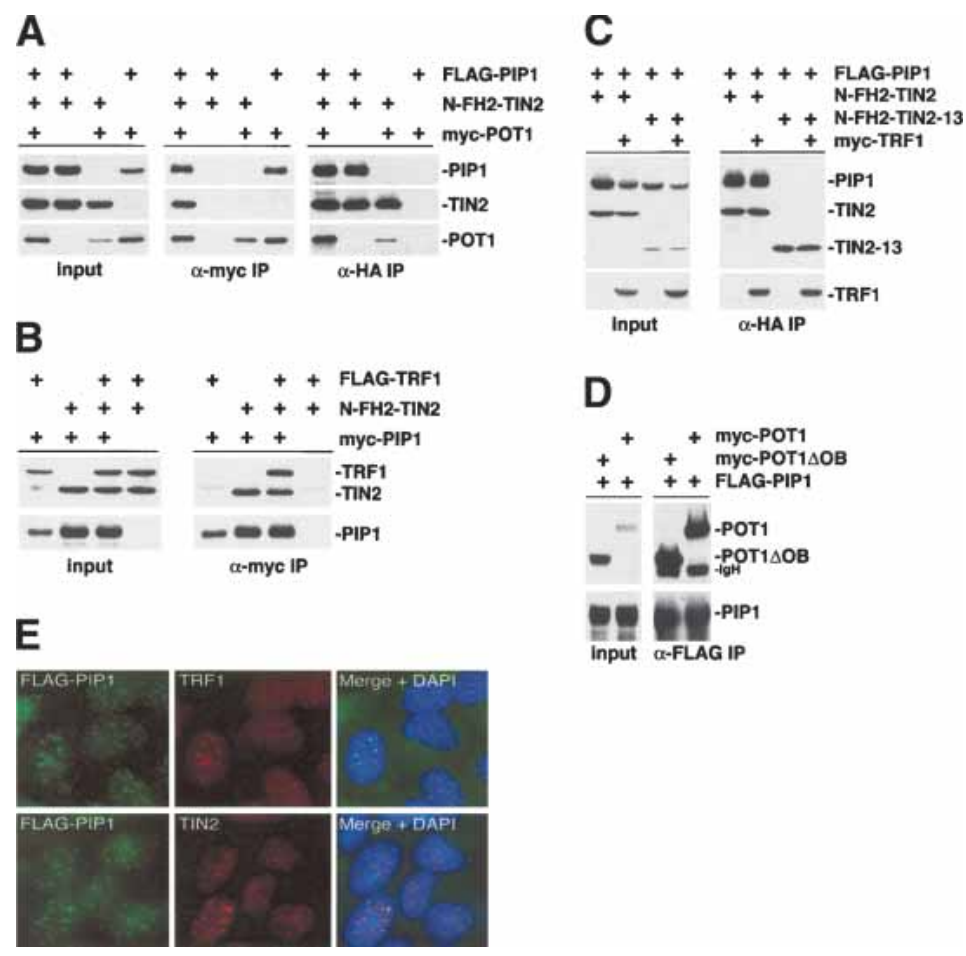

Figure 1. Interactions of PIP1 with TIN2 and POT1 and localization of PIP1 to telomeres. (A) Co-IP of PIP1 with POT1 and TIN2. Epitope-tagged constructs were transfected into 293T cells in various combinations (top) and subjected to coIP using antibodies to the myc or HA tags. Protein expression levels are shown in input lysates. Immunoblots were done with the myc antibody to detect POT1 and the Flag antibody to detect PIP1 and TIN2. (B) Co-IP of PIP1 with TIN2 but not TRF1. The indicated constructs were cotransfected into 293T cells, and the anti-myc (PIP1) IPs were analyzed for the presence of PIP1 (myc antibody), TRF1, and TIN2 (Flag antibody for both). (C) PIP1 does not interact with TIN2-13. N-FH2-TIN2 or N-FH2-TIN2-13 were coexpressed with Flag-PIP1 and mycTRF1 as indicated. Proteins recovered in the anti-HA immunoprecipitate were analyzed by immunoblotting. TRF1 was detected with the myc antibody, and PIP1, TIN2, and TIN2-13 were detected with the Flag antibody. $(D)$ PIP1 interacts with POT1 $\triangle \mathrm{OB}$. Flag-PIP1 was coexpressed with myc-tagged full-length POT1 or POT1 $\triangle \mathrm{OB}$. IPs were performed with Flag-antibody and analyzed by immunoblotting with antibodies to the epitope tags. $(E)$ Telomeric localization of exogenous PIP1. IF of HeLa1.2.11 cells expressing Flag-PIP1 from a retrovirus (pLPC). PIP1 was detected with anti-Flag (M2; green). TRF1 (top) and TIN2 (bottom) were detected with Abs 371 and 865, respectively (red). DNA was stained with DAPI (shown in the merged panel). 

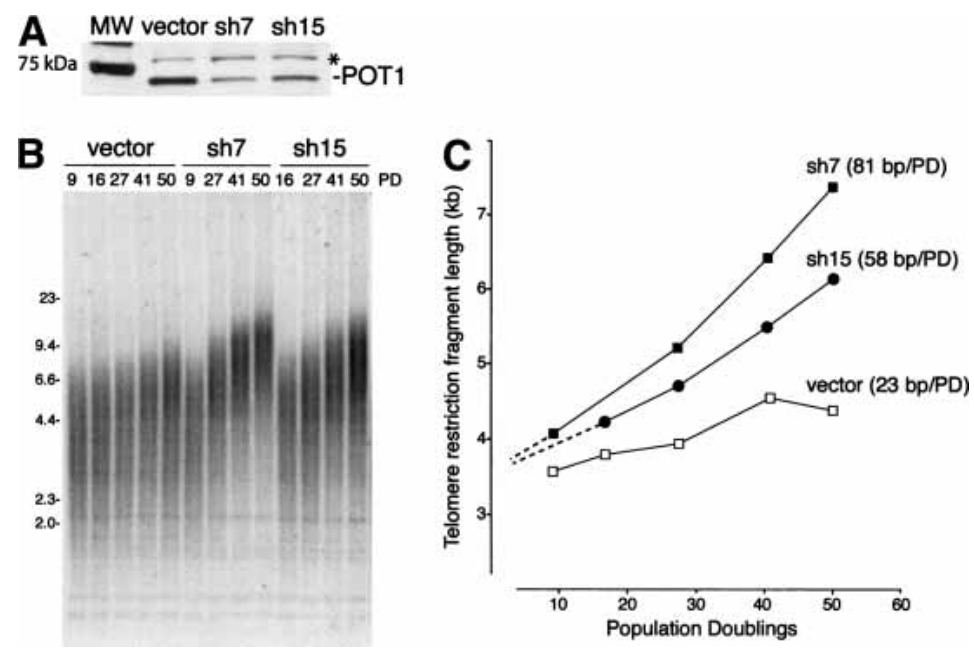

Figure 2. Telomere elongation induced by RNAi-mediated reduction of POT1. (A) Immunoblot of HTC75 cells expressing the indicated shRNAs (vector control represents cells infected with the empty virus) at PD50 postselection. POT1 was detected with Ab 978 as described previously (Loayza and de Lange 2003). The asterisk marks a cross-reacting band that serves as a loading control. $(B)$ Telomeric restriction fragment blot of the cell lines shown in $A$ performed at the indicated population doublings (PDs). The molecular mass in kilobases of HindIII-digested $\lambda$ DNA fragments is shown on the left. (C) Graph of the mean telomeric restriction fragment length of the indicated cell lines plotted versus PD. Elongation rates of the telomeres are indicated to the right.

with the patterns of TRF1 and TIN2 (Fig. 1E). Collectively, these results indicate that PIP1 is a telomeric protein that interacts with TIN2 and can recruit POT1 to the TRF1 complex.
myc-PIP1 (Fig. 3A). The corresponding shRNAs induced telomere elongation in HTC75 cells, whereas expression of Flag-PIP1 had no effect on telomere length (Fig. 3B,C). The simplest interpretation of these results is that PIP1 regulates telomere length through recruitment of POT1 to the TRF1 complex. Because TRF1, TIN2, and POT1 do not affect telomere length in telomerase-negative cells (Kim et al. 1999; Karlseder et al. 2002; Loayza and de Lange 2003), it is likely that the TRF1/TIN2/PIP1/POT1 pathway controls telomere length through an effect on telomerase.

\section{Conclusion}

We have identified a new component of the telomeric TRF1 protein complex, PIP1, which interacts with TIN2 and POT1. TRF1 and TIN2 were previously shown to be negative regulators of telomere length (van Steensel and de Lange 1997; Kim et al. 1999; Ancelin et al. 2002; Ye and de Lange 2004). The RNAi experiments reported here show that PIP1 and POT1 are also negative regulators of telomere length. Together with previous studies on POT1, these data fit a model (Fig. 4) in which the duplex telomeric repeats can recruit POT1 through a direct interaction of the telomeric DNA-binding factor TRF1 with TIN2, the interaction of TIN2 with PIP1, and finally the interaction of PIP1 with POT1. This set of interactions explains why long telomeres, which bind more TRF1, also contain more POT1 (Loayza and de Lange 2003). The presence of POT1 on longer telomeres is proposed to be responsible for the final outcome of this

\section{Telomere elongation in cells with reduced POT1 or PIP1 levels}

POT1 was proposed to be a negative regulator of telomere length based on the rapid telomere elongation phenotype of the POT $1 \Delta \mathrm{OB}$ mutant (Loayza and de Lange 2003). To corroborate the role of POT1 in telomere length control further, we used RNAi to diminish the expression of POT1 in HTC75 cells, a subclone of the telomerase positive fibrosarcoma cell line HT1080, widely used for telomere length regulation studies (van Steensel and de Lange 1997). Two retrovirally expressed shRNAs directed against POT1 resulted in a significant reduction of POT1 expression compared with the vector control (Fig. 2A). There was no detectable change in the growth rate of cells infected with POT1 shRNAs (data not shown), indicating that HTC75 cells tolerate the reduced levels of POT1. Consistent with this, POT1 levels remained stably reduced for $>50$ population doublings (data not shown). Telomere length analysis showed significant telomere elongation in these cells (Fig. 2B,C), confirming that POT1 acts as a negative regulator of telomere length.

A similar approach was taken to determine the telomere length phenotype of reduced PIP1 levels. Two siRNAs directed against PIP1 resulted in significant reduction of PIP1 levels in a transient transfection assay of HTC75 cells expressing
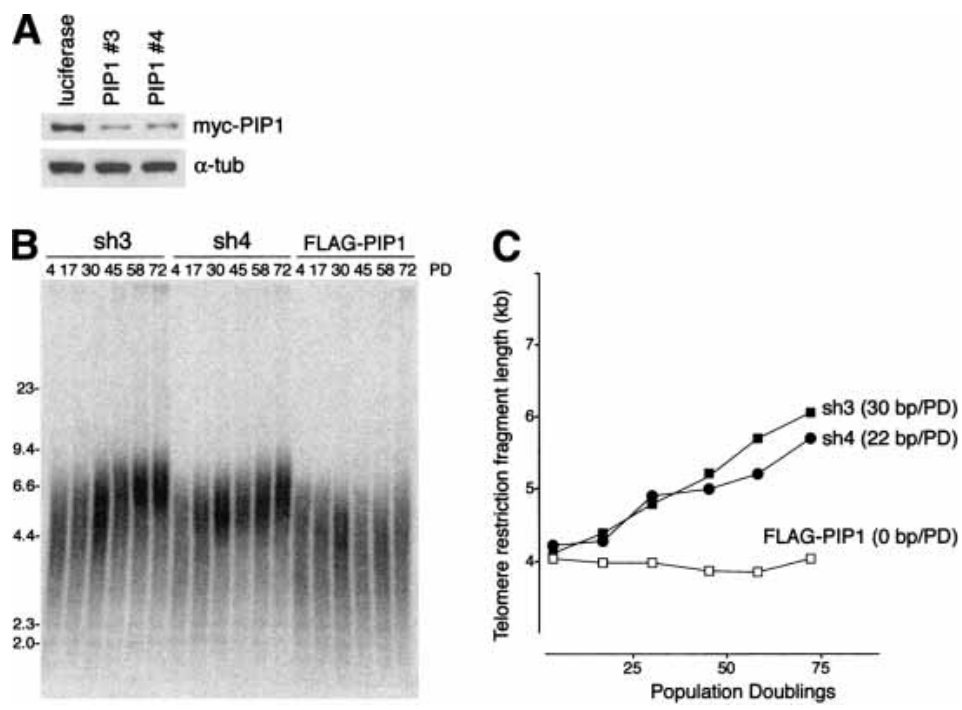

Figure 3. Telomere elongation induced by RNAi-mediated reduction of PIP1. (A) HTC75 cells expressing myc-tagged PIP1 were transfected with siRNA targeting luciferase (control) and two independent siRNAs targeting PIP1 (\#3 and \#4). Myc-PIP1 levels were detected by immunoblotting using 9E10 (Oncogene) with $\alpha$-tubulin as loading control. (B) Telomere lengthening caused by PIP1 knockdown. Telomeric restriction fragment blot of the HTC75 cells infected with PIP1 shRNA retroviruses or Flag-tagged PIP1. Population doublings (PDs) are indicated above the lanes. The molecular mass in kilobases of HindIII-digested $\lambda$ DNA fragments is shown on the left. (C) Graph of the mean telomeric restriction fragment length of the indicated cell lines plotted versus PD. Elongation rates of the telomeres are indicated to the right. 
A

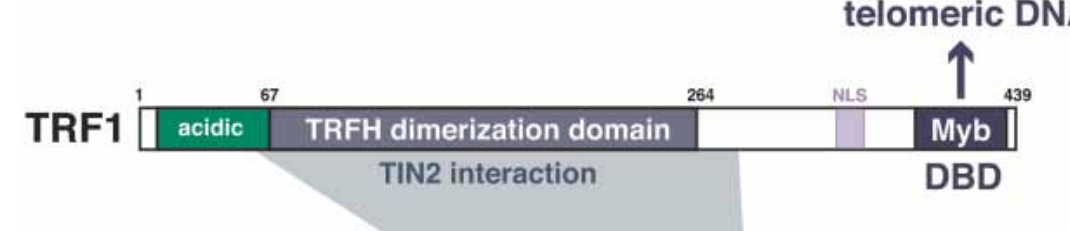

TIN2

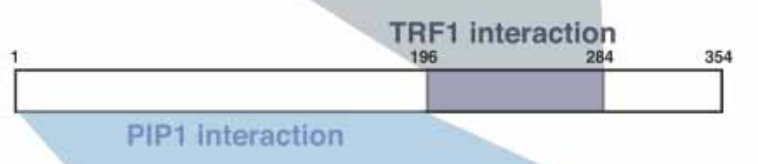

PIP1

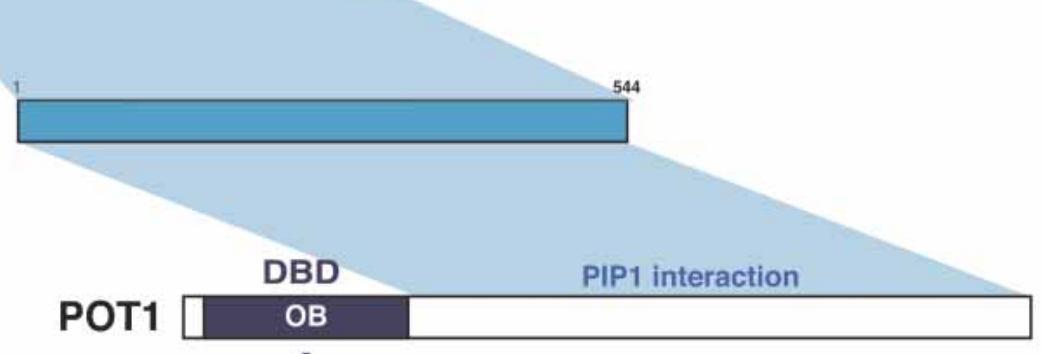

$\downarrow$

single-stranded telomeric DNA
B
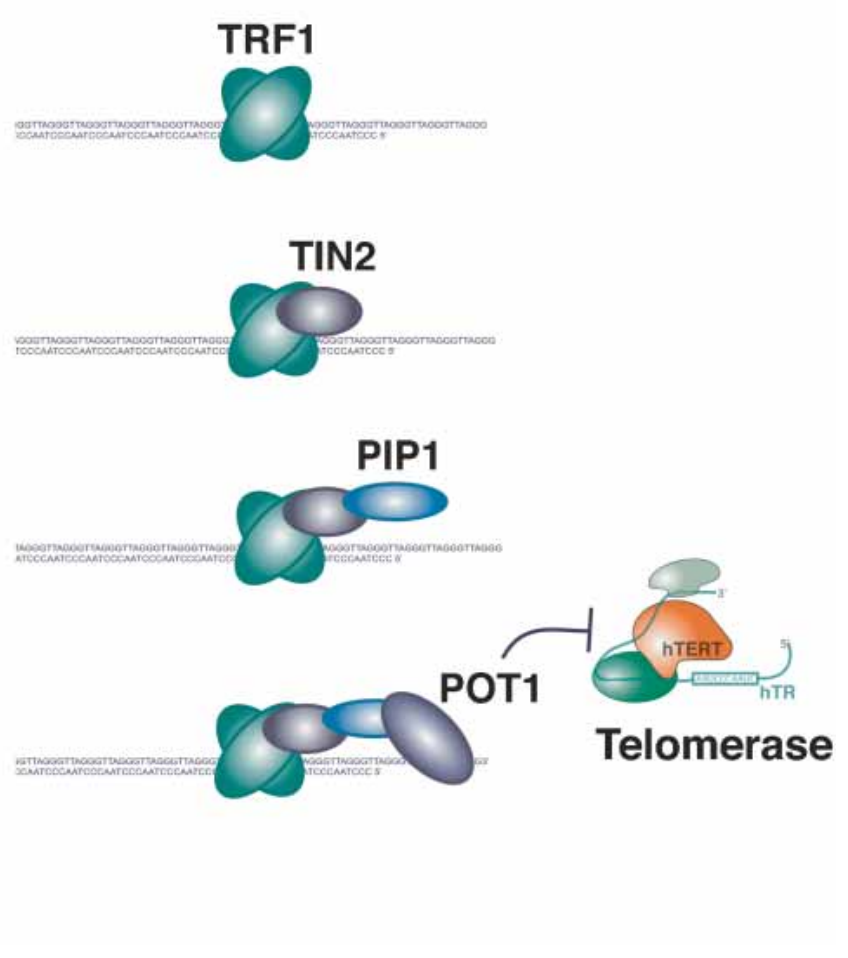

Figure 4. Summary of the TRF1-TIN2-PIP1-POT1 telomere length regulation pathway. (A) Components of the TRF1 complex and their interactions with DNA and/or proteins. The PIP1 interaction with TIN2 has not been mapped in detail but is abolished by deletion of the indicated domain. (B) Schematic of POT1-mediated telomere length regulation, emphasizing the protein interactions leading to recruitment of POT1. It is possible (likely) that some or all of the components of the TRF1 complex are associated with each other prior to the binding of TRF1 to telomeric DNA. It is not known whether POT1 can bind to single-stranded DNA while interacting with PIP1. 
pathway, the cis-inhibition of telomerase. Because deletion of the N-terminal OB fold of POT1 abrogates telomere length control, POT1's ability to bind singlestranded DNA appears crucial for telomere length homeostasis. Our data suggest that the main role of PIP1 at telomeres is to recruit POT1 to the telomeric chromatin. However, it is not excluded that PIP1 also serves other functions in the telomeric complex. In this regard, TIN2 has been shown to play at least two roles in the TRF1 complex. On the one hand, as shown here, TIN2 is a recruitment module that ensures the binding of PIP1 and POT1 to telomeres. On the other hand, recent data indicate that TIN2 also functions in stabilizing TRF1 on telomeric DNA by blocking the action of tankyrase 1 (Ye and de Lange 2004).

\section{Materials and methods}

\section{HeLaS3 clone expressing tagged TIN2 and TRF1}

Two retroviral vectors based on pLPC were generated by introducing a Flag-HA-HA tag (FH2) in a N-terminal or C-terminal position (details available on request). TIN2 and TRF1 cDNA were PCR-amplified and cloned separately into either the N- or C-terminally tagged constructs and sequenced. These constructs were transfected into amphotrophic Phoenix cells using standard calcium phosphate method and the resulting retroviral stocks were used to infect semiadherent HeLaS3 cells. Clones expressing tagged TRF1 and TIN2 were selected with puromycin $(2 \mu \mathrm{g} / \mathrm{mL})$ and isolated using cloning cylinders. The efficiency of $\mathrm{N}$ - and C-terminally tagged TRF1 and TIN2 in forming complex with endogenous TIN2 and TRF1, as well as tankyrase 1, were tested by coIP. N-FH2-TIN2 and C-FH2-TRF1 were found to interact with other components of the TRF1 complex more efficiently. To obtain a single HeLaS3 clone expressing both tagged TRF1 and TIN2, N-FH2-TIN2 was tranduced into a C-FH2-TRF1-expressing HeLaS3 clone by superinfection without additional drug selection under conditions of $\sim 100 \%$ infection. The resulting infectants were ring-cloned. The final cell line was a HeLa S3 clone that expressed both tagged proteins at the level $\sim 5$ - to 10-fold above that of the endogenous proteins. Sequential $\mathrm{KCl}$ extraction of chromatin proteins was performed as described previously (Ye and de Lange 2004). For purification of the TRF1-TIN2 complex, HeLaS3 cells were grown in suspension culture $(20 \mathrm{~L})$ at $37^{\circ} \mathrm{C}$ to a density between 0.9 and $1.2 \times 10^{6} / \mathrm{mL}$. Cell harvest, extraction of nuclear proteins, sequential binding to affinity matrix, and peptide elutions were performed according to published procedures (Ogawa et al. 2002), except that commercial affinity resins were used (anti-Flag M2-resin from Sigma, and anti-HA 3F10 resin from Roche).

\section{Mass spectrometry}

Eluted proteins were separated by SDS-PAGE $(4 \%-20 \%$ gradient; Invitrogen). The entire gel lane was sliced into $292-\mathrm{mm}$ pieces. Proteins in each gel piece were subjected to trypsin digestion. The resulting peptides were extracted and the proteins identified using a combination of two different mass spectrometers. First, tryptic mass maps of proteins from each gel piece were obtained using an in-house constructed MALDIQqTOF mass spectrometer; and second, fragmentation spectra of all the discernable tryptic peptides were obtained using an in-house-constructed MALDI-ion trap mass spectrometer (Krutchinsky et al. 2000, 2001). Accurate masses of the tryptic peptides and the masses of their fragments were used to identify proteins in each gel piece with the computer search engine XProteo (Chao Zhang; http://www.xproteo.com).

\section{Yeast two-hybrid analysis}

Fusions with the LexA DNA-binding domain or with the GAL4 activation domain were generated by cloning PCR-amplified fragments from full-length cDNA-containing plasmids (details available on request) using pBTM116 and pACT2. The TRF1, TRF2, and hRap1 fusions with LexA were described previously (Bianchi et al. 1997; Li et al. 2000). The POT1-LexA construct was generated by fusing the third amino acid of POT1 to the LexA DNA-binding domain using the BamHI site of pBTM116. TIN2 was fused to LexA at the second amino acid of TIN2 using the EcoRI site of pBTM116. PIP1 was fused to the GAL4 activation domain using the BamHI site in pACT2. The two-hybrid tests were performed in the yeast strain L40 (MATa his3L200 trp1-901 leu2-3,112 ade2 LYS2::(lexAop) $)_{4}$-HIS3 URA3::(1exAop) $)_{8}$-lacZ). Quantitative $\beta$-galactosidase activities were performed as described by the manufacturer's protocols (Clontech). The average value for three independent transformants with the indicated bait-prey combinations is indicated in Table 1 , and the values for each individual transformant diverged by $<10 \%$ from the average.

\section{Co-IP from 2937 cells}

Human $293 \mathrm{~T}$ cells $\left(5-6 \times 10^{6} / 10 \mathrm{~cm}\right.$ dish $)$ were plated and transfected 20-24 h later using the calcium phosphate coprecipitation method and 10-20 $\mu \mathrm{g}$ of plasmid DNA per dish. Medium was changed after $12 \mathrm{~h}$, and cells were harvested $24-30 \mathrm{~h}$ after transfection. Cells were dislodged from the dish by flushing with cold PBS, collected by centrifugation, and lysed in ice-cold buffer $(50 \mathrm{mM}$ Tris- $\mathrm{HCl}$ at $\mathrm{pH} 7.4,20 \%$ glycerol, $1 \mathrm{mM}$ EDTA, $150 \mathrm{mM} \mathrm{NaCl}, 0.5 \%$ Triton X-100, 0.02\% SDS, $1 \mathrm{mM}$ dithiothreitol [DTT], $2 \mathrm{mM}$ phenylmethylsulfonyl fluoride [PMSF], $1 \mu \mathrm{g} / \mathrm{mL}$ aprotinin, $10 \mu \mathrm{g} / \mathrm{mL}$ pepstatin, and $1 \mu \mathrm{g} / \mathrm{mL}$ leupeptin). After $5 \mathrm{~min}$ on ice, $5 \mathrm{M}$ $\mathrm{NaCl}$ was added to bring the final $[\mathrm{NaCl}]$ to $400 \mathrm{mM}$. After another $5 \mathrm{~min}$ on ice, an equal volume of ice-cold water was added and thoroughly mixed before immediate centrifugation in a microfuge (14 krpm, $10 \mathrm{~min}$ ). Supernatants were collected and used directly for IP. Lysates prepared from one 10-cm dish were mixed with monoclonal antibodies (per IP: anti-Flag/M2 [Sigma], $5 \mu \mathrm{g}$; of anti-HA, 3F10 [Roche], $1.2 \mu \mathrm{g}$; anti-myc, $9 \mathrm{E} 10$ [Oncogene], 0.6-1.0 $\mathrm{\mu g}$ for $5-6 \mathrm{~h}$ at $4^{\circ} \mathrm{C}$ while rocking on a nutator). During the final hour, $30 \mu \mathrm{L}$ of protein G-Sepharose beads (settled volume) was added to each tube (beads were preblocked o/n with $10 \%$ BSA in PBS). Beads were washed three times with 1:1 diluted lysis buffer, proteins were eluted with Laemmli loading buffer, and analyzed by SDSPAGE and immunoblotting.

\section{RNA interference}

POT1 levels were stably reduced upon expression of two shRNAs from the pSUPERIOR retroviral vector (OligoEngine). The sequences of the shRNA targets are GTACTAGAAGCCTATCTCA for sh7 and GATAT TGTTCGCTTTCACA for sh15. Cells were grown in the presence of doxycyclin $(250 \mathrm{ng} / \mathrm{mL})$. Two shRNA retroviral constructs were made to target PIP1 mRNA using pSUPER.retro vector (OligoEngine). The two PIP1 targeting sequences in siRNA and shRNA were AAGCTCTAT GACTGCCTTG for \#3 and CCAGCCATCTGCTCAGCCC for \#4. Retroviruses were produced from amphotrophic Phoenix cells and used to transduce HTC75 cells followed by puromycin selection.

To test PIP1 RNAi targeting, siRNAs were transfected with oligofectamine (Invitrogen) into HTC75 cells expressing myc-PIP1. Specifically, $2 \times 10^{5}$ cells/well were inoculated in six-well plates. After 18-20 h, cells were subjected to two sequential transfections, separated by a $24-\mathrm{h}$ interval. Cells were harvested by trypsin-EDTA, counted, and lysed in Laemmli buffer. After shearing, lysates from $10^{5}$ cells were separated by SDS-PAGE and analyzed by Western blotting using anti-myc (9E10) antibody (Oncogene).

\section{Telomere length analysis}

Genomic DNA was isolated from HTC75 cultures grown as described previously (van Steensel and de Lange 1997) except that Phase Lock Gel tubes (Eppendorf) were used for phenol extraction. DNA was digested with AluI and MboI, quantified using Hoechst fluorimetry, separated on a $0.7 \%$ agarose gel, and transferred to a Hybond-N membrane for hybridization using an $\left[\alpha-{ }^{32} \mathrm{P}\right] \mathrm{dCTP}$ Klenow-labeled 800-bp telomeric DNA probe from pSP73Styl1 (de Lange et al. 1990; de Lange 1992). Blots were exposed to PhosphorImager screens, and mean telomeric restriction fragment lengths were determined using ImageQuan. Rates of telomere elongation were calculated by linear regression.

\section{Indirect immunofluorescence}

Cells grown on glass coverslips were fixed for $10 \mathrm{~min}$ at room temperature with PBS containing $2 \%$ paraformaldehyde, and permeabilized for 10 $\mathrm{min}$ in PBS $+0.5 \%$ NP- 40 . Cells were preblocked for at least 30 min with PBG (PBS $+0.2 \%$ cold water fish gelatin and $0.5 \% \mathrm{BSA}$ ) prior to incubation with primary antibody $\left(2 \mathrm{~h}\right.$ at room temperature or o/n at $\left.4^{\circ} \mathrm{C}\right)$. 
Ye et al.

Purified rabbit polyclonal anti-TIN2 (\#865), anti-TRF1 (\#371), and monoclonal anti-Flag (M2; Sigma) were used as primary antibodies. Rhodamine- or fluorescein-conjugated donkey anti-rabbit or anti-mouse antibodies (Jackson Laboratory) were used as secondary antibodies at dilutions recommended by the manufacturer. Bleedthrough controls were performed by leaving out one of the primary antibodies.

\section{Acknowledgments}

We thank H. Ogawa and Y. Nakatani (Harvard) for providing the protocol for the purification of tagged protein complexes. R. Roeder (RU) is thanked for providing facilities to grow HeLa cells. Members of the de Lange lab are thanked for helpful discussion and comments on this manuscript. This work was supported by grants from the NCI and Burroughs Welcome to T.d.L., by an NIH grant to B.T.C. (RR00862), by a Career Development Award from the NCI (K08 CA93604) to J.Z.Y., and by a grant to J.Z.Y. from the Translational \& Integrative Medicine Research Fund from Sloan-Kettering Cancer Institute. D.H. is supported by the Rockefeller University Graduate Program. D.L. is supported by an NCI training grant (T32 CA09673-26A1).

The publication costs of this article were defrayed in part by payment of page charges. This article must therefore be hereby marked "advertisement" in accordance with 18 USC section 1734 solely to indicate this fact.

\section{Note added in proof}

PIP1 was also identified as part of the TRF1 complex by Songyang and colleagues (Liu et al. 2004).

\section{References}

Ancelin, K., Brunori, M., Bauwens, S., Koering, C.E., Brun, C., Ricoul, M., Pommier, J.P., Sabatier, L., and Gilson, E. 2002. Targeting assay to study the cis functions of human telomeric proteins: Evidence for inhibition of telomerase by TRF1 and for activation of telomere degradation by TRF2. Mol. Cell. Biol. 22: 3474-3487.

Baumann, P. and Cech, T.R. 2001. Pot1, the putative telomere end-binding protein in fission yeast and humans. Science 292: 1171-1175.

Bianchi, A., Smith, S., Chong, L., Elias, P., and de Lange, T. 1997. TRF1 is a dimer and bends telomeric DNA. EMBO J. 16: 1785-1794.

Chong, L., van Steensel, B., Broccoli, D., Erdjument-Bromage, H., Hanish, J., Tempst, P., and de Lange, T. 1995. A human telomeric protein. Science 270: 1663-1667.

de Lange, T. 1992. Human telomeres are attached to the nuclear matrix. EMBO J. 11: 717-724.

de Lange, T., Shiue, L., Myers, R.M., Cox, D.R., Naylor, S.L., Killery, A.M., and Varmus, H.E. 1990. Structure and variability of human chromosome ends. Mol. Cell. Biol. 10: 518-527.

Karlseder, J., Smogorzewska, A., and de Lange, T. 2002. Senescence induced by altered telomere state, not telomere loss. Science 295: 24462449 .

Kim, S.H., Kaminker, P., and Campisi, J. 1999. TIN2, a new regulator of telomere length in human cells. Nat. Genet. 23: 405-412.

Krutchinsky, A.N., Zhang, W., and Chait, B.T. 2000. Rapidly switchable matrix-assisted laser desorption/ionization and electrospray quadrupole-time-of-flight mass spectrometry for protein identification. $J$. Am. Soc. Mass Spectrom. 11: 493-504.

Krutchinsky, A.N., Kalkum, M., and Chait, B.T. 2001. Automatic identification of proteins with a MALDI-quadrupole ion trap mass spectrometer. Anal. Chem. 73: 5066-5077.

Li, B., Oestreich, S., and de Lange, T. 2000. Identification of human Rap1: Implications for telomere evolution. Cell 101: 471-483.

Liu, D., Safari, A., O'Conner, M.S., Chan, D.W., Laegeler, A., Qin, L., and Songyang, Z. 2004. PTOP is a novel POT1 interacting protein that regulates POT1 telomeric localization. Nat. Cell Biol. (in press).

Loayza, D. and de Lange, T. 2003. POT1 as a terminal transducer of TRF1 telomere length control. Nature 424: 1013-1018.

Loayza, D., Parsons, H., Donigian, J., Hoke, K., and de Lange, T. 2004. DNA binding features of human POT1: A nonamer 5'-TAGGGT TAG-3' minimal binding site, sequence specificity, and internal binding to multimeric sites. J. Biol. Chem. 279: 13241-13248.

Marcand, S., Gilson, E., and Shore, D. 1997. A protein-counting mechanism for telomere length regulation in yeast. Science 275: 986-990.

Ogawa, H., Ishiguro, K., Gaubatz, S., Livingston, D.M., and Nakatani, Y. 2002. A complex with chromatin modifiers that occupies E2F- and Myc-responsive genes in G0 cells. Science 296: 1132-1136.

Smogorzewska, A. and de Lange, T. 2004. Regulation of telomerase by telomeric proteins. Ann. Rev. Biochem. 73: 177-208.

Smogorzewska, A., van Steensel, B., Bianchi, A., Oelmann, S., Schaefer, M.R., Schnapp, G., and de Lange, T. 2000. Control of human telomere length by TRF1 and TRF2. Mol. Cell. Biol. 20: 1659-1668.

van Steensel, B. and de Lange, T. 1997. Control of telomere length by the human telomeric protein TRF1. Nature 385: 740-743.

Ye, J.Z.-S. and de Lange, T. 2004. TIN2 is a tankyrase 1 PARP-modulator in the TRF1 telomere length control complex. Nat. Genet. 36: 618623. 


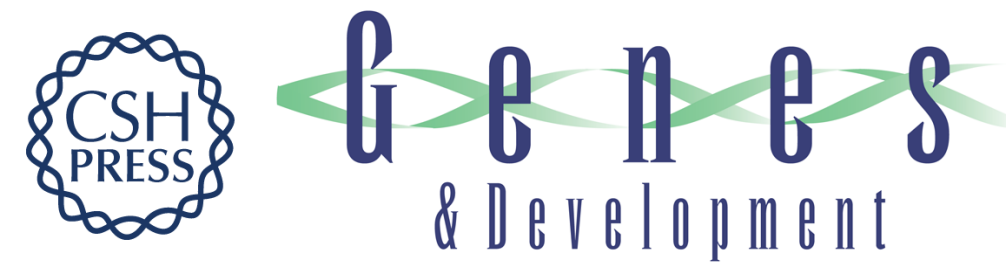

\section{POT1-interacting protein PIP1: a telomere length regulator that recruits POT1 to the TIN2/TRF1 complex}

Jeffrey Zheng-Sheng Ye, Dirk Hockemeyer, Andrew N. Krutchinsky, et al.

Genes Dev. 2004, 18:

Access the most recent version at doi:10.1101/gad.1215404

\section{Supplemental http://genesdev.cshlp.org/content/suppl/2004/07/02/1215404.DC1 Material}

References This article cites 19 articles, 10 of which can be accessed free at: http://genesdev.cshlp.org/content/18/14/1649.full.html\#ref-list-1

\section{License}

Email Alerting

Receive free email alerts when new articles cite this article - sign up in the box at the top Service

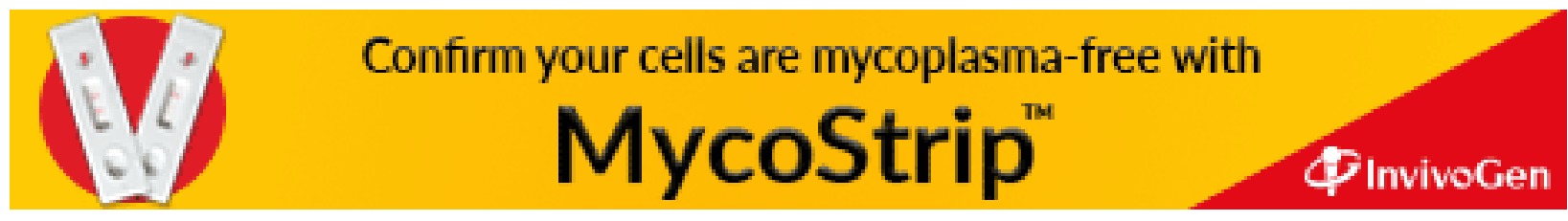

